\title{
Special issue on spatial and temporal database management
}

\author{
Christophe Claramunt ${ }^{1} \cdot$ Markus Schneider $^{2}$ • \\ Raymond Chi-Wing Wong ${ }^{3}$
}

Published online: 22 August 2017

(C) Springer Science+Business Media, LLC 2017

This special issue contains six contributions selected as best papers from the 14th International Symposium on Spatial and Temporal Databases (SSTD) held in Hong Kong in August 2015. SSTD is an established series of events that continuously demonstrate how much spatial and temporal data are still popular and active research domains of interest, with still many research issues to address and application areas to explore. Over the past few years the range of topics addressed has evolved, with the emergence of big spatial and temporal data, location-based services, environmental and urban sensors to mention a few examples, thus generating a lot of new research problems and novel methods still to be explored in order to deliver appropriate data manipulation and interfaces at the user level.

The selected papers published in this special issue are representatives of the recent developments of spatial-temporal data management. One popular form of mobile data is trajectories each representing a sequence of sampled points of a moving object. The paper by Shuyao Qi et al. (Snapshot and Continuous Points-based Trajectory Search) performs data analysis on these trajectories. On the other hand, shortest distance computation is one important topic in the literature. The paper by Alexandros Efentakis et al. (Hub Labels on the database for large-scale graphs with the COLD framework) studies how to use hub labels for shortest-distance computation. Recently, privacy has attracted a lot of attention due to its importance. This privacy issue was studied by Mihai Maruseac et al. (Privacy-Preserving Detection of Anomalous Phenomena in Crowdsourced Environmental Sensing using FineGrained Weighted Voting). The paper entitled 'Knowledge Extraction from Crowdsourced Data for the Enrichment of Road Networks' written by G. Josse et al. studied how to enrich the information of road networks by extracting knowledge from crowdsourced data. The paper

Raymond Chi-Wing Wong

raywong@cse.ust.hk

1 Naval Academy Research Institute, Lanvéoc, France

2 University of Florida, Gainesville, FL, USA

3 The Hong Kong University of Science and Technology, Hong Kong, Hong Kong 
entitled 'Approximate UV computation based on space decomposition' written by K. A. Schmid, A. Zufle, T. Emrich, M. Renz and R. Chang introduces a Voronoi decomposition to manage uncertain data. The objective is to apply a hierarchical access method in both data and object spaces. The experiments show good processing times and then provide a promising computational solution for neighboring queries. Another paper 'Discovering Non-compliant Window Co-occurrence Patterns' whose co-authors are R. Y. Ali, V. M. V. Gunturi, A. J. Kotz, E. Eftelioglu, S. Shekhar and W. F. Northrop searches for temporal signatures amongst a set of spatio-temporal trajectories. A bi-directional pruning approach is suggested and experimented in the context of real data extracted from engine measurement data.

We hope that these papers will inspire researchers to further conduct research in the literature of spatio-temporal data management. 